\title{
PERFIL LIPÍDICO DE OBESOS ANTES E APÓS A DERIVAÇÃO GÁSTRICA A FOBI-CAPELLA
}

\section{LIPID PROFILE OF OBESE BEFORE AND AFTER FOBI-CAPELLA GASTRIC BYPASS}

\author{
Eduardo Nacur Silva, ACBC-MG'; Marcelo Dias Sanches²
}

\begin{abstract}
RESUMO: Objetivo: Este estudo analisa de forma retrospectiva o perfil lipídico de pacientes submetidos à derivação gástrica a Fobi-Capella, na Santa Casa de Belo Horizonte de setembro de 1999 a setembro de 2003. Método: Foram avaliados os protocolos de acompanhamento de 143 pacientes, analisando os valores de colesterol total (CT), colesterol associado à lipoproteína de alta densidade (HDL), colesterol associado à lipoproteína de baixa densidade (LDL), triglicérides (TG) e o índice de massa corporal (IMC) no pré-operatório e um ano após a operação. A presença de dislipidemia foi definida como alteração em pelo menos um dos exames. Foi feita a comparação entre os valores do pré-operatório com os do pós-operatório. Resultados: Os valores séricos médios do CT, HDL, LDL e TG foram de 201,7 mg\%, 47,9 mg\%, 122,6 mg\% e 153,7\% respectivamente. Não houve diferença nestes valores quando estratificados pelo IMC. A prevalência de dislipidemia foi de $61,5 \%$ no pré-operatório. Após um ano da operação, houve queda de $12,5 \%$ no CT, $14,3 \%$ no LDL, $29,5 \%$ nos TG e aumento de 18,5\% no HDL. Ocorreu normalização em 73,9\% dos pacientes que apresentavam pelo menos uma das dosagens alterada. Conclusão: A prevalência da dislipidemia foi elevada nos pacientes submetidos à derivação gástrica a Fobi-Capella. Ocorreu melhora dos parâmetros avaliados do perfil lipídico após um ano da operação (Rev. Col. Bras. Cir. 2006; 33(2): 91-95).
\end{abstract}

Descritores: Obesidade mórbida; Derivação gástrica; Hiperlipidemia; Colesterol; Triglicérideos; Período pós-operatório.

\section{INTRODUÇÃO}

A alteração dos níveis séricos dos lípides, seja do colesterol, de suas frações e dos triglicérides, é fator importante para o aparecimento de doença cardiovascular (DCV) de origem aterosclerótica ${ }^{1}$. A relação direta entre DCV e elevação do nível sérico do colesterol total (CT) e do colesterol associado à lipoproteína de baixa densidade (LDL) foi demonstrada em estudos com acompanhamento em longo pra$\mathrm{ZO}^{2,3,4}$. Da mesma forma, a relação inversa entre o colesterol associado à lipoproteína de alta densidade (HDL) e DCV também já foi demonstrada. A discussão sobre a participação dos triglicérides (TG) na aterogênese permanece ainda em pauta e a dosagem sérica de rotina é justificada pela freqüente vinculação a DCV aterosclerótica e pela associação com outros distúrbios metabólicos. ${ }^{1}$ Além disso, a normalização dos níveis séricos dos lipídes, seja reduzindo o CT, o LDL e os TG ou elevando o HDL, reduziu de modo significativo o aparecimento de DCV de origem aterosclerótica ${ }^{4}$.

A dislipidemia, ou seja, a alteração dos níveis séricos dos lípides, é uma condição freqüentemente associada à obesidade, sendo considerada como co-morbidade desta afecção ${ }^{5,6,7}$. O grau de obesidade pode ser definido pelo índice de massa corporal (IMC) que é calculado dividindo-se o peso em quilos pela altura em metros elevada ao quadrado. Quando a obesidade supera o nível de $40 \mathrm{~kg} / \mathrm{m}^{2}$ é considerada como obesidade mórbida (OM).A falha dos tratamentos clínicos para a OM é superior a $90,0 \%$, o que estimulou a busca por outras alternativas de tratamento destes pacientes. No Brasil, a técnica da derivação gástrica a Fobi-Capella foi introduzida por Garrido e é a mais utilizada no nosso meio para tratamento cirúrgico da OM. Consiste na associação da gastroplastia vertical com colocação de anel de silicone associada à derivação gastrojejunal em y de Roux ${ }^{8-13}$.

Deste modo, o presente estudo tem como objetivo estudar a prevalência da dislipidemia nos obesos submetidos à derivação gástrica a Fobi-Capella e avaliar o efeito desta técnica no perfil lipídico destes pacientes.

Este estudo foi aprovado pela Comissão de Ética em Pesquisa da Santa Casa de Belo Horizonte.

\section{MÉTODO}

Este é um estudo retrospectivo realizado na Santa Casa de Belo Horizonte. Foram avaliados os protocolos de 143 pacientes submetidos à derivação gástrica a Fobi-Capella entre setembro de 1999 e setembro de 2003 . Nenhum paciente utilizou medicamento que sabidamente alterasse a dosagem sérica dos lípides. Todos foram operados pelo mesmo cirurgião. A rotina de acompanhamento dos pacientes submetidos a esta operação incluiu retornos no $1^{\circ}, 3^{\circ}, 6^{\circ}$ e $12^{\circ}$ meses pósoperatórios para avaliação clínica e laboratorial.

Foram considerados como valores de referência da normalidade o padrão definido pela sociedade brasileira de cardiologia: dosagem sérica do CT até $200 \mathrm{mg} \%$, do HDL maior ou igual a $40 \mathrm{mg} \%$, do LDL até $130 \mathrm{mg} \%$ e dos TG até $200 \mathrm{mg} \%$.

1. Cirurgião Geral da Santa Casa de Belo Horizonte - MG

2. Professor Adjunto-Doutor do Departamento de Cirurgia da Faculdade de Medicina da UFMG.

Recebido em 20/10/2005

Aceito para publicação em 22/12/2005

Conflito de interesses: nenhum

Aceito para publicação em:

Trabalho realizado na Santa Casa de Belo Horizonte - MG. 
Foi considerado com portador de dislipidemia o paciente que apresentasse pelo menos um valor alterado e considerado como normal àquele que tivesse todos os valores dentro dos limites de referência. Os valores médios do CT, HDL, LDL e TG foram estratificados de acordo com o IMC, sendo considerados os valores dos pacientes com IMC entre 35 e $39,9 \mathrm{~kg} / \mathrm{m}^{2}$, entre 40 e $49,9 \mathrm{~kg} / \mathrm{m}^{2}$ e maiores ou iguais a $50 \mathrm{~kg} / \mathrm{m}^{2}$.

Foi realizada a comparação entre as dosagens séricas de CT, do HDL, do LDL, TG e a \% do excesso de IMC perdido entre os valores pré-operatórios e após um ano da operação.

Foi testada a correlação entre a porcentagem do excesso de IMC perdido e a alteração no perfil lipídico. O cálculo desta porcentagem foi feito com a seguinte fórmula:

$$
\% \text { excesso de IMC perdido }=100-\frac{[\text { IMCfinal - 25 }}{\text { IMCpré }-25} \times 100
$$

\section{RESULTADOS}

Trinta e seis pacientes $(25,2 \%)$ eram do sexo masculino e 107 (74,8\%) do feminino. Aidade média foi de 38,8 anos (17-64 anos), o peso médio foi de $122,7 \mathrm{~kg}(85-266 \mathrm{~kg})$ e o IMC médio de $45,1 \mathrm{~kg} / \mathrm{m}^{2}\left(35,2-86,9 \mathrm{~kg} / \mathrm{m}^{2}\right)$.

Os valores séricos do CT, HDL, LDL e TG foram em média de 201,7mg\%, 47,9mg\%, 122,6 mg\% e 153,7 mg\%, respectivamente.O perfil lipídico dos 143 pacientes demonstrou uma prevalência variável de alterações. No pré-operatório, 69 pacientes $(48,3 \%)$ apresentavam o $\mathrm{CT}$ alterado. O HDL estava diminuído em 39 pacientes $(27,3 \%)$, o LDL estava elevado em 54 pacientes $(37,8 \%)$ e os TG elevados em 31 pacientes $(21,7 \%)$. Oitenta e oito pacientes $(61,5 \%)$ apresentaram pelo menos um exame alterado, enquanto em 55 pacientes $(38,5 \%)$ os resultados de todos os exames eram normais.

Os valores do CT, HDL, LDL e TG foram separados de acordo com estratificação do IMC (IMC $<40,40 £$ IMC $<50$ e IMC $\geq 50 \mathrm{~kg} / \mathrm{m}^{2}$ ). Nesta estratificação, apenas o valor médio dos TG é maior comparando o terceiro grupo com o segundo, porém a média destes dois grupos não difere se comparados com os pacientes com IMC menor que $40 \mathrm{~kg} / \mathrm{m}^{2}$. (Tabela 1)

A taxa de retorno aos 12 meses foi de $60,8 \%$ (87 pacientes). Destes, 70 pacientes $(49,0 \%)$ possuíam os resultados de todos os exames deste estudo (CT, HDL, LDL e TG).

Após um ano de pós-operatório, ocorreu queda significativa dos níveis séricos do CT, LDL e TG e aumento do HDL. A porcentagem de queda do CT, LDL e dos TG foi de $12,5 \%, 14,3 \%$ e $29,5 \%$ respectivamente. O aumento do HDL foi de $18,5 \%$. (Tabela 2 e 3 )

Ocorreu normalização em $34(73,9 \%)$ dos 46 pacientes que tinham pelo menos um exame alterado. Porém ocorreu alteração no exame de um paciente $(4,2 \%)$ do grupo de 24 que não apresentavam dislipidemia no pré-operatório. No pós-operatório, a prevalência de dislipidemia foi de $18,6 \%$, sendo encontrado pelo menos um dos exames alterados em 13 dos 70 pacientes avaliados. Após um ano, ocorreu normalização dos valores do CT em 78,8\% dos pacientes que apresentavam o CT elevado no pré-operatório. Mesmo entre os que não houve normalização, houve diminuição dos valores absolutos. O nível sérico do HDL era normal em 55 pacientes $(78,6 \%)$. Entre os 15 pacientes que tinham o HDL alterado houve normalização em 11 (77,3\%). Quanto ao nível sérico do LDL, 45 pacientes $(64,3 \%)$ tinham o valor pré-operatório dentro dos valores de referência. Dos que estavam com os exames alterados, 25 pacientes, em 21 (84,0\%) ocorreu normalização dos mesmos. Entre os 70 pacientes avaliados após um ano da operação, 58 (82,9\%) tinham o nível sérico dos TG normal no pré-operatório. Ele normalizou em 75,0\% dos pacientes cujo nível era elevado no pré-operatório, com queda nos demais com exames alterados.

Não houve correlação significativa entre a porcentagem do IMC perdido e as alterações ocorridas nos valores séricos dos lípides. (Tabela 4)

\begin{tabular}{|c|c|c|c|c|c|c|c|}
\hline \multirow[b]{2}{*}{ Variável } & \multirow[b]{2}{*}{ IMC } & \multirow[b]{2}{*}{$\mathbf{n}$} & \multicolumn{4}{|c|}{ Medidas Descritivas } & \multirow[b]{2}{*}{ p } \\
\hline & & & Mínimo & Máximo & Média & d.p. & \\
\hline \multirow{3}{*}{ CT mg\% } & $\underline{\mathrm{IMC}<40}$ & 26 & 152,0 & 283,0 & 204,9 & 36,3 & \\
\hline & $40 \leq \mathrm{IMC}<50$ & 91 & 125,0 & 357,0 & 200,6 & 42,3 & 0,897 \\
\hline & $\geq 50$ & 26 & 121,0 & 263,0 & 202,0 & 40,9 & $1=2=3$ \\
\hline \multirow{3}{*}{ HDL mg\% } & $\underline{\mathrm{IMC}<40}$ & 26 & 29,0 & 85,0 & 49,8 & 15,9 & \\
\hline & $40 \leq \mathrm{IMC}<50$ & 91 & 22,0 & 89,0 & 47,2 & 12,9 & 0,671 \\
\hline & $\geq 50$ & 26 & 31,0 & 82,0 & 48,4 & 11,9 & $1=2=3$ \\
\hline \multirow{3}{*}{ LDL mg\% } & $\underline{\mathrm{IMC}}<40$ & 26 & 60,0 & 178,0 & 119,4 & 32,3 & \\
\hline & $40 \leq \mathrm{IMC}<50$ & 91 & 53,0 & 259,0 & 124,5 & 38,2 & 0,724 \\
\hline & $\geq 50$ & 26 & 37,0 & 173,0 & 119,3 & 37,4 & $1=2=3$ \\
\hline \multirow{3}{*}{ TGmg\% } & $\underline{\mathrm{IMC}}<40$ & 26 & 59,0 & 445,0 & 163,9 & 91,9 & \\
\hline & $40 \leq \mathrm{IMC}<50$ & 91 & 39,0 & 417,0 & 142,1 & 67,3 & 0,032 \\
\hline & $\geq 50$ & 26 & 59,0 & 465,0 & 184,4 & 81,9 & $2<1=3$ \\
\hline
\end{tabular}

Tabela 1 - Análise descritiva e comparativa dos lípides estratificados pelo IMC.

Nota: $O$ valor de p na tabela refere-se ao teste da Análise de Variância com 1 fator (IMC). 
Tabela 2 - Variação do CT, HDL, LDL e TG após um ano da operação em relação ao pré-operatório (\%).

\begin{tabular}{llcccc}
\hline & & \multicolumn{4}{c}{ Medidas descritivas } \\
\cline { 3 - 6 } Variável & $\mathbf{n}$ & Mínimo & Máximo & Média & d.p. \\
\hline CT & 70 & $+22,3$ & $-38,6$ & $-12,5$ & 12,5 \\
HDL & 70 & $-23,0$ & $+90,3$ & $+18,5$ & 25,8 \\
LDL & 70 & $+51,9$ & $-53,3$ & $-14,3$ & 24,9 \\
TG & 70 & $+57,5$ & $-75,1$ & $-29,5$ & 26,3 \\
\hline
\end{tabular}

Cálculos das variáveis:

$C T \%=[(C T$ pós-operatório $-C T$ pré-operatório $) / C T$ pré-opera tório] $\times 100$

$H D L \%=[(H D L$ pós-operatório $-H D L$ pré-operatório $) / H D L$ pré-operatório] $\times 100$

$L D L \%=[(L D L$ pós-operatório $-L D L$ pré-operatório $) / L D L$ préoperatório] $\times 100$

$T G \%=[(T G$ pós-operatório - TG pré-operatório $) /$ TG pré-operatório] $\times 100$

\section{DISCUSSÃO}

Os valores do IMC, idade e distribuição por sexo encontrados neste estudo estão de acordo com a maioria dos estudos publicados com pacientes submetidos a tratamento cirúrgico da OM.

Entretanto, a prevalência da dislipidemia em obesos mórbidos é muito variável. Isto pode ser explicado em parte por diferentes critérios adotados como valores de referência da normalidade. A Sociedade Brasileira de Cardiologia, atra- vés do seu departamento de aterosclerose, recomenda valores semelhantes aos norte-americanos para a dosagem sérica do CT, do HDL, do LDL e dos TG na III Diretrizes Brasileiras sobre dislipidemias ${ }^{14}$. Como o objetivo deste estudo não incluiu a análise destes fatores de risco, optamos pelo valor normal do LDL até $130 \mathrm{mg} \%$, pois este valor é o mais utilizado atualmente.

A prevalência de dislipidemia é muito variável entre os pacientes com OM, oscilando entre $19,0 \%$ a $82,9 \%{ }^{15-17}$. Em alguns estudos considera-se dislipidemia a alteração em apenas um dos valores medidos, seja CT, HDL, LDL ou TG e em outros somente quando ocorre combinação de alterações. No Brasil, dois estudos envolvendo pacientes com OM, um no Sudeste e outro no Nordeste, encontraram uma prevalência de dislipidemia que variou entre $40,0 \%$ e $66,7 \%{ }^{5,18}$. É possível que as diferenças encontradas entre estes estudos ocorram em função do grupo racial e dos hábitos alimentares. Os valores de referência dos dois estudos também foram diferentes. Neste estudo classificamos como dislipidemia a alteração em pelo menos uma das variáveis estudadas (CT, HDL, LDL ou TG). Assim pudemos verificar o efeito da derivação gástrica a Fobi-Capella nas dosagens séricas dos lípides no pós-operatório, seja no conjunto dos exames ou em cada um isoladamente.

A estratificação por IMC não é padrão nos estudos que avaliaram a influência das diversas técnicas de operações bariátricas no perfil lipídico. No nosso estudo não houve diferença entre os grupos, quando se separou pelo IMC, em relação ao CT e suas frações, HDL e LDL. Quanto aos TG, a diferença entre o grupo de pacientes com IMC maior que $50 \mathrm{~kg} / \mathrm{m}^{2}$

Tabela 3 - Comparação entre as medidas do pré-operatório e após um ano da operação.

\begin{tabular}{|c|c|c|c|c|c|c|c|}
\hline \multirow[b]{2}{*}{ Variável } & \multirow[b]{2}{*}{ Tratamento } & \multirow[b]{2}{*}{$\mathbf{n}$} & \multicolumn{4}{|c|}{ Medidas descritivas } & \multirow[b]{2}{*}{ p } \\
\hline & & & Mínimo & Máximo & Média & d.p. & \\
\hline \multirow{3}{*}{$\mathrm{CT} \mathrm{mg \%}$} & Pré & 70 & 125,0 & 357,7 & 201,3 & 41,1 & \\
\hline & & & & & & & 0,0001 \\
\hline & Pós & 70 & 79,0 & 278,0 & 173,9 & 31,2 & Pré > Pós \\
\hline \multirow{3}{*}{ HDL mg\% } & Pré & 70 & 24,0 & 89,0 & 47,8 & 13,4 & \\
\hline & & & & & & & 0,0001 \\
\hline & Pós & 70 & 30,0 & 99,0 & 54,9 & 12,9 & Pré < Pós \\
\hline \multirow{3}{*}{ LDL mg\% } & Pré & 70 & 37,0 & 259,0 & 120,6 & 37,6 & \\
\hline & & & & & & & 0,0001 \\
\hline & Pós & 70 & 33,0 & 183,0 & 98,9 & 27,9 & Pré > Pós \\
\hline \multirow{3}{*}{$\begin{array}{l}\text { Pré } \\
\text { TGmg\% }\end{array}$} & 70 & 48,0 & 445,0 & 155,1 & 75,8 & & \\
\hline & & & & & & & 0,0001 \\
\hline & Pós & 70 & 26,0 & 280,0 & 102,5 & 47,1 & Pré > Pós \\
\hline \multirow{3}{*}{$\mathrm{IMC} \mathrm{kg} / \mathrm{m}^{2}$} & Pré & 70 & 35,2 & 61,5 & 44,4 & 6,1 & \\
\hline & & & & & & & 0,0001 \\
\hline & Pós & 70 & 20,7 & 50,0 & 29,9 & 5,2 & Pré > Pós \\
\hline
\end{tabular}

Nota: $O$ valor de p na tabela refere-se ao teste t de Student para amostras pareadas 
Tabela 4 - Análise de correlação entre porcentagem de excesso de IMC perdido e perfil lipídico.

\begin{tabular}{lll}
\hline Variável & $\mathbf{r}$ & $\mathbf{p}$ \\
\hline CT \% & 0,14 & 0,2359 \\
HDL \% & 0,14 & 0,2489 \\
LDL \% & 0,23 & 0,0586 \\
TG\% & $-0,05$ & 0,6886 \\
\hline
\end{tabular}

Nota: valor de p na tabela refere-se ao teste da Análise de Correlação de Pearson.

$r \rightarrow$ Coeficiente de correlação.

e os com IMC entre $40 \mathrm{~kg} / \mathrm{m}^{2}$ e $50 \mathrm{~kg} / \mathrm{m}^{2}$ não pode ser explicada pelo peso aumentado do primeiro grupo, pois os pacientes com IMC menor que $40 \mathrm{~kg} / \mathrm{m}^{2}$ não apresentavam diferença significativa comparando com o grupo de maior IMC. Um aumento no número de pacientes estudados poderá mostrar a real prevalência desta alteração.

O mecanismo da mudança no perfil lipídico após o emagrecimento cirurgicamente induzido ainda não foi totalmente explicado. A perda de peso não parece ser isoladamente o fator exclusivo, pois a correlação entre esta e as alterações encontradas não são uniformes. A influencia da dieta hipocalórica, em alguns casos hipolipídica, parece ser fator importante, mas também não exclusivo. Isto sugere que o excesso de peso não seja a única causa de dislipidemia nos pacientes com OM, e sim parte de um complexo de alterações metabólicas, dietéticas e de outros fatores que resultam nas anormalidades lipídicas associadas à obesidade ${ }^{19}$. No nosso estudo houve melhora significativa dos níveis séricos de CT, LDL, HDL e TG após um ano de pós-operatório, com aumento do HDL e diminuição dos demais. Estas alterações não são constantes após este tipo de operação.

A maioria dos estudos mostra apenas variação dos níveis séricos dos lípides, seja queda ou elevação. Em estudos que enfatizam a correção dos valores alterados no préoperatório só há referência aos resultados positivos. Os negativos são referidos como não resolução do problema.

Brolin et al. ${ }^{20}$ avaliaram 130 pacientes submetidos a derivação gástrica, encontraram prevalência de hiperlipidemia de $19 \%$, com normalização em $67,6 \%$, melhora em $20,6 \%$ e sem resposta em $11,8 \%$. Outra referência mostrou melhora em $96 \%$ dos indivíduos com dislipidemia, com resolução em 63,0\% dos pacientes com hipercolesterolemia e em $57 \%$ dos com hipertrigliceridemia submetidos a derivação gástrica em Y de Roux $^{15}$. Em estudo realizado no Brasil, a normalização dos níveis séricos do CT ocorreu em 51,7\%, melhora em 44,8\% e sem alterações em $3,5 \%$.

Frigg et al. ${ }^{22}$ apesar de relatarem melhora significativa dos casos com dislipidemia, fazem referência específica aos casos de piora. Eles encontraram taxa de conversão de exames normais para alterados de $15 \%$ após um ano e de $8 \%$ após dois anos da colocação de banda gástrica ajustável.

No nosso estudo houve resolução da dislipidemia em 73,9\% dos casos, valor semelhante ao encontrado em outros estudos. Ocorreu apenas um caso $(4,2 \%)$ de piora do perfil lipídico no pós-operatório.

Todas estas alterações já foram questionadas se seriam apenas secundárias à mudança radical da dieta ou se fariam parte de uma alteração metabólica produzida pela operação ${ }^{19}$. O fato é que a mudança da dieta, por si só pode levar a alterações semelhantes, principalmente em relação ao HDL e TG. E além disto, a redução do peso dá condições aos pacientes de terem uma vida mais ativa que também contribui para estas alterações ${ }^{23}$. Apesar das variações encontradas envolverem inter-relações não completamente compreendidas, a influência da dieta hipocalórica já foi bem definida ${ }^{19}$.

Uma redução entre 5 e $10 \%$ do peso corporal já é suficiente para reduzir os fatores de risco para $\mathrm{DCV}^{15}$. Este fato pode explicar, em parte, a falta de correlação entre a perda de peso e as alterações encontradas neste estudo, pois a menor perda do excesso de IMC após um ano foi de 31,6\%.

Gleysteen et al. ${ }^{24}$ não encontraram correlação entre perda de peso e variações no perfil lipídico, após cirurgias de derivação gástrica. Entretanto, Brolin et al. ${ }^{20}$ encontraram esta correlação quando analisaram os exames pós-operatórios dos pacientes que já os tinham alterados no pré-operatório. Eles explicam o fato de conseguirem demonstrar esta correlação por avaliar pacientes com nível sérico de lípides inicialmente mais alto.

Podemos concluir que a prevalência pré-operatória de dislipidemia nos pacientes submetidos à derivação gástrica a Fobi-Capella foi elevada. Não houve relação entre a dosagem sérica do CT, do HDL, do LDLe dos TG com o IMC.Houve melhora significativa do perfil lipídico após um ano da derivação gástrica a Fobi-Capella.

\begin{abstract}
Background: The direct relationship between elevated cholesterol and atherosclerosis is known for a long time. Dyslipidemia is frequent in obese patients. Surgery for treatment of morbid obesity aims to loose weight and correction of co-morbidities. This study analyses the lipid profile of obese before and one year after gastric bypass. Methods: Charts of 143 patients were reviewed evaluating the levels of cholesterol (TC), cholesterol linked to high-density lipoprotein (HDL), cholesterol linked to low-density lipoprotein $(L D L)$, trigliycerides $(T G)$ and body mass index (BMI) pre-operatively and one year after the operation. Results: The levels of TC, HDL, LDL and TG were $201.7 \mathrm{mg} \%, 47.9 \mathrm{mg} \%, 122.6 \mathrm{mg} \%$ and $153.7 \%$ respectively. One year after the operation, the levels of TC dropped 12.5\%, LDL 14.3\%, TG 29.5\% and HDL raised 18.5\%. The lipid profile normalized in $73.9 \%$ of patients. Conclusion: The pre-operative prevalence of dyslipidemia is high in morbid obese patients. There is a significant improvement in the lipid profile one year after the gastric bypass.
\end{abstract}

Key words: Obesity, morbid; Gastric bypass; Hyperlipidemia: Cholesterol; Tryglicerides; Post-operative period. 


\section{REFERÊNCIAS}

1- Gianini SD. Aterosclerose e dislipidemias. $1^{\text {a }}$ ed. São Paulo: BG Editora e Produções Culturais Ltda; 1998.

2- Anderson KM, Castelli WP, Levy D. Cholesterol and mortality. JAMA. 1987; 257(16):2176-80.

3- Castelli WP, Garrison RJ, Wilson PWF, Abbott RD, Kalousdian $\mathrm{S}$, Kannel WB. Incidence of coronary heart disease and lipoprotein cholesterol levels. The Framingham study. JAMA. 1986; 256(20):2835-8.

4- Manninen V, Ello MO, Frick MH, Haapa K, Heinonen OP, Heinsalmi $\mathrm{P}$, et al. Lipid alterations and decline in the incidence of coronary heart disease in the Helsinki Heart Study. JAMA. 1988; 260(5):641-51.

5- Cercato C, Mancini MC, Arguello AMC, Passos VQ, Villares SMF, Halpern A. Systemic hypertension, diabetes mellitus, and dislipidemia in relation to body mass index: evaluation of a brasilian population. Rev Hosp Clin Fac Med São Paulo. 2004; 59(3):1138.

6- Kral JG. Morbid obesity and related health risks. Ann Inter Med. 1985; 103(6):1043-7

7- Stubbs RS, Wickremesekera SK. Insulin resistance in severely obese links with metabolic co-morbidities. Obes Surg. 2002; 12(3):343-8.

8- Capella JF, Capella RF, Mandac H, Nath P. Vertical banded gastroplasty - gastric bypass: preliminary report. Obes Surg. 1991; 1(4):389-95.

9- Capella JF, Capella RF. The weight reduction operation choice: vertical banded gastroplasty or gastric bypass. Am J Surg. 1996; 171(1):74-9.

10- Fobi MAL, Lee H, Fleming AW. The surgical technique of the banded Roux-en-Y gastric bypass. J Obes and Weight Regulation. 1989; 8(1):99-102.

11- Fobi MAL. Vertical banded gastroplasty vs. gastric bypass: 10 years follow-up. Obes Surg. 1993; 3(2):161-4.

12- Garrido Jr AB, Berti LV, Oliveira MR, Elias AA, Matielli JD, Matsuda M, Gama-Rodrigues JJ. A operação de Capella por laparotomia. In: Atualização em cirurgia do aparelho digestivo e em coloproctologia. São Paulo: Departamento de Gastroenterologia da Faculdade de Medicina da Universidade de São Paulo; 2004. p. 223-32.

13- Garrido Jr AB, Oliveira MR, Berti LV, Elias AA, Pareja JC, Matsuda M, Gama-Rodrigues JJ. Derivações gastrojejunais. In: Garrido Jr AB, Ferraz EM, Barroso FL, Marchesini JB, Szego T. Cirurgia da obesidade. São Paulo: Atheneu ; 2002. p. 155-61.

14- Resumo das III diretrizes brasileiras sobre dislipidemias e diretriz de prevenção de aterosclerose do Departamento de
Aterosclerose da Sociedade Brasileira de Cardiologia. Arq Bras Cardiol. 2001; 77(supl 3): 4-8.

15- Diniz MTC, Diniz MFHS, Barros HG. Evolução das comorbidades no pós-operatório de cirurgia bariátrica. In: SavassiRocha PR, Coelho LGV, Diniz MTC, Nunes TA. Tópicos em gastroenterologia 13. Obesidade e urgências gastroenterológicas. Rio de Janeiro: MEDSI; 2003. p. 141-54.

16- Ferraz EM, Arruda PCL, Bacelar TC, Ferraz AAB, Albuquerque AC, Leão CS. Tratamento cirúrgico da obesidade mórbida. Rev Col Bras Cir. 2003; 30(2):98-105.

17- Giusti V, Suter M, Héraief E, Gaillard RC, Burckhardt P. Effects of laparoscopic gastric banding on body composition, metabolic profile and nutricional status of obese women: 12-months followup. Obes Surg. 2004; 14(2):239-45.

18- Porto MCV, Brito IC, Calfa ADF, Amoras M, Villela NB, Araújo LMB. Perfil do obeso classe III do ambulatório de um hospital universitário de Salvador, Bahia. Arq Bras Endocrinol Metab. 2002; 46(6):668-73.

19- Kelly TM, Jones SB. Changes in serum lipids after gastric bypass surgery. Lack of relationship to weight loss. Int J Obes. 1986; 10(6): 443-52.

20- Brolin RE, Kenler HA, Clemow LP, Kasnetz KA, Ebert EC, Greenfield DP, et al. Gastric reduction surgery for morbid obesity. New Jersey Med. 1987; 84(7):481-8.

21- Valesi AC, Mali Jr J, Brito EM, Marson AC. Gastroplastia vertical com bandagem em Y-de-Roux: análise de resultados. Rev Col Bras Cir. 2004; 31(1):49-56.

22- Frigg A, Peterli R, Peters T, Ackermann C, Tondelli P. Reduction in co-morbidities 4 years after laparoscopic adjustable gastric banding. Obes Surg. 2004; 14(2):216-23.

23- Bronstein MD. Exercício físico e obesidade. Rev Soc Cardiol Estado de São Paulo. 1996; 6(1):111-6.

24- Gleysteen JJ, Barboriak JJ. Improvement in heart disease risk factors after gastric bypass. Arch Surg. 1983; 118(6):681-4.

Como citar este artigo:

Silva EN, Sanches MD. Perfil lipídico de obesos antes e após a derivação gástrica a Fobi-Capella. Rev Col Bras. [serial on the Internet] 2006 Mar-Apr; 33(2). Available from URL: http:// www.scielo.br/rcbc

Endereço para correspondência:

Eduardo Nacur Silva

Rua Santa Rita Durão, 1194 / apto. 1002

CEP: 30140-111 - Belo Horizonte - MG

E-mail: ensbh@terra.com.br 